\title{
Sharp Bounds on the Spectral Radius of Nonnegative Matrices and Comparison to the Frobenius' Bounds
}

\author{
Maria Adam \\ Dept. of Computer Science \\ \& Biomedical Informatics \\ University of Thessaly \\ Lamia, Greece \\ madam@dib.uth.gr
}

\author{
Nicholas Assimakis \\ General Department \\ National and Kapodistrian \\ University of Athens \\ Athens, Greece \\ nasimakis@uoa.gr
}

\author{
Fotis Babouklis \\ Dept. of Computer Science \\ \& Biomedical Informatics \\ University of Thessaly \\ Lamia, Greece \\ fbampouklis@uth.gr
}

Received: March 9, 2020, Revised: July 24, 2020. Accepted: July 30, 2020. Published July 31, 2020.

\begin{abstract}
In this paper, a new upper bound and a new lower bound for the spectral radius of a nonnegative matrix are proved by using similarity transformations. These bounds depend only on the elements of the nonnegative matrix and its row sums and are compared to the well-established upper and lower Frobenius' bounds. The proposed bounds are always sharper or equal to the Frobenius' bounds. The conditions under which the new bounds are sharper than the Frobenius' ones are determined. Illustrative examples are also provided in order to highlight the sharpness of the proposed bounds in comparison with the Frobenius' bounds. An application to linear invariant discrete-time nonnegative systems is given and the stability of the systems is investigated. The proposed bounds are computed with complexity $O\left(\mathbf{n}^{2}\right)$.
\end{abstract}

Keywords-Nonnegative matrix, Spectral radius, Row sum, Stability, Algorithm, Complexity

\section{INTRODUCTION}

Let $M_{n}(\mathbb{R})$ be the algebra of $n \times n$ real matrices. We refer to $A=\left(a_{i j}\right)_{i, j=1}^{n} \in M_{n}(\mathbb{R})$ as a nonnegative or a positive matrix, when each $a_{i j} \geq 0$ or $a_{i j}>0$, respectively, denoted by writing $A \geq 0$ or $A>0$. The matrix $A \in M_{n}(\mathbb{R})$ is called irreducible if and only if $(I+A)^{n-1}>0$. We also define the spectral radius of $A$ by $\rho(A)=\max \{|\lambda|: \lambda \in \sigma(A)\}$, where $\sigma(A)$ denotes the spectrum of $A$, that is, the set of eigenvalues of $A$. In case of $A$ is symmetric $\rho(A)$ is the largest eigenvalue of $A$.

For $1 \leq i \leq n$, the $i$-th row sum of $A$, denoted by $r_{i}(A)$, is

$$
r_{i}(A)=\sum_{j=1}^{n} a_{i j}
$$

In recent decades, the problem of bounding the largest eigenvalue in modulus of a nonnegative matrix has attracted the interest of many researchers (see, [1-4, 7-8, 14-20] and the references therein), since Perron-Frobenius theory plays an important role in the mathematical fields of dynamical systems, cryptography, control and graph theory (see, e.g. [1$2,4,7-8,12-20])$. For example, the topological entropy, which is one of the main invariants of a topological dynamical system telling us how chaotic the system is, can often be computed as a logarithm of the spectral radius of a certain nonnegative matrix [20]. Furthermore, the analysis of discrete-time positive switched systems is based on the Perron-Frobenius theory and the graph-theoretic arguments (see, [3, 9, 13] and the references therein). The stability issues of linear invariant discrete-time nonnegative systems are connected to the spectral radius of a matrix of the system (see, $[3,5-6,13,17]$ and the references therein).

The well-known Perron-Frobenius theory has investigated the existence of positive eigenvalues of nonnegative matrices $[11,16]$. In particular, Perron has proved that $\rho(A)$ is a positive and simple eigenvalue of $A>0$ [11, Theorem 8.2.11] and Frobenius has generalized Perron's statement for nonnegative and irreducible matrices [11, Theorem 8.4.4] giving lower and upper bounds for the spectral radius.

Algebraic methods for the computation of the bounds of the spectral radius of a nonnegative matrix have been presented in $[1,4,8,14-15,18]$ giving applications in the spectral radii of the various matrices of a graph or a digraph, including the signless Laplacian spectral radius, the distance spectral radius and the distance signless Laplacian spectral radius, the spectral radius of the reciprocal distance matrix (see, $[1,8,14-15,18]$ and the references therein). The bounds of the spectral radius, which provided in the aforementioned studies, depend on the size, the elements, the row sum and various average row sums of the nonnegative matrix and their computation is more complicated than the computation of the largest eigenvalue of the matrix.

Moreover, apart from the familiar power method, other numerical algorithms have been also constructed and implemented for locating the spectrum of matrices as in [7, $13,21]$. However, the proposed methods are only valid on the limited class of diagonalizable matrices or on irreducible nonnegative matrices, whose spectral radius has been proved to be a simple eigenvalue. The computation of the spectral radius is achieved with complexity $\mathrm{O}\left(\mathrm{n}^{3}\right)$. Hence, often requires a good estimate of the largest eigenvalue from an upper and a lower bound, which are computed with complexity $\mathrm{O}\left(\mathrm{n}^{2}\right)$, where $\mathrm{n}$ is the size of the nonnegative matrix

The novelty of this paper concerns (a) the determination with simple calculations of new upper and lower bounds for the spectral radius of a nonnegative matrix, which are always 
sharper or equal to the Frobenius' bounds and (b) the presentation of the complexity required for the proposed bounds computation.

The paper is organized as follows. In Section II, new formulas are presented bounding the spectral radius of any nonnegative matrix providing bounds, which depend on the elements of the matrix. The conditions under which the new bounds are sharper than the Frobenius' ones are investigated. The spectral radius of the special nonnegative matrices is formulated and suitable example illustrate the results provided in the paper. In Section III, an application in stability of a single-input single-output (SISO) linear discrete time systems is presented and the stability of the systems is investigated. A linear system's stability check criterion based on the proposed upper bound is derived. In Section IV, the algorithms for computing the upper and lower bounds of the spectral radius of a nonnegative matrix are summarized and their complexity is analyzed. Finally, Section V summarizes the conclusions.

\section{BOUNDS FOR THE SPECTRAL RADIUS}

The next lemmas demonstrate well-established bounds for $\rho(A)$, which have been formulated by Frobenius and since they are used in our arguments, they are stated here.

Lemma 1. ([11, Corollary 8.1.20, Theorem 8.1.22]) Let $A=\left(a_{i j}\right)_{i, j=1}^{n} \in M_{n}(\mathbb{R}) \quad, \quad A \geq 0 \quad$ with $i$-th row sum $r_{i}(A), i=1, \ldots, n$. Then,

$$
\begin{gathered}
\max _{1 \leq i \leq n}\left\{a_{i i}\right\} \leq \rho(A) \\
r_{\lambda}(A) \equiv \min _{1 \leq i \leq n}\left\{r_{i}(A)\right\} \leq \rho(A) \leq \max _{1 \leq i \leq n}\left\{r_{i}(A)\right\} \equiv r_{\mu}(A)
\end{gathered}
$$

Moreover, if $A$ is irreducible, then equality can hold on either side of (3) if and only if $r_{1}(A)=r_{2}(A)=\cdots=r_{n}(A)$.

Definition 2. For a $\mathrm{n}$ onnegative matrix $A=\left(a_{i j}\right)_{i, j=1}^{n} \in M_{n}(\mathbb{R})$ consider the quantities

$$
Z_{i}=\left\{\begin{array}{cc}
\frac{-b_{i}+\sqrt{b_{i}^{2}+4 a_{i \lambda}\left(r_{\lambda}(A)-a_{\lambda \lambda}\right)}}{2\left(r_{\lambda}(A)-a_{\lambda \lambda}\right)}, & \text { if } r_{\lambda}(A)>a_{\lambda \lambda} \\
i, & \text { if } r_{\lambda}(A)=a_{\lambda \lambda}
\end{array}\right.
$$

and

$$
w_{j}=\left\{\begin{array}{cc}
\frac{-c_{j}+\sqrt{c_{j}^{2}+4 a_{j \mu}\left(r_{\mu}(A)-a_{\mu \mu}\right)}}{2\left(r_{\mu}(A)-a_{\mu \mu}\right)}, & \text { if } r_{\mu}(A)>a_{\mu \mu} \\
1 / j, & \text { if } r_{\mu}(A)=a_{\mu \mu}
\end{array}\right.
$$

where $r_{\lambda}(A), r_{\mu}(A)$ are denoted in (3) and $b_{i}=a_{i \lambda}+a_{\lambda \lambda}-r_{i}(A), c_{j}=a_{j \mu}+a_{\mu \mu}-r_{j}(A)$, for $1 \leq i, j \leq n$, and $i \neq \lambda, j \neq \mu$.

Remark 3. Here note that, if $A \geq 0$, then $r_{i}(A)=a_{i i} \Leftrightarrow a_{i j}=0, \quad$ for all $1 \leq j \leq n, \quad i \neq j, \quad$ and $a_{i i} \in \sigma(A)$.

i) Thus, $z_{i}$ in $(4 b)$ are defined for the matrices, where the minimum row sum appears to $\lambda$ - th row and all off-diagonal elements of the $\lambda$-th row are equal to zero and then $a_{\lambda \lambda} \in \sigma(A)$. ii) Similarly, $w_{j}$ in $(5 b)$ are defined for the matrices, where the maximum row sum appears to $\mu$ - th row and all offdiagonal elements of the $\mu-$ th row are equal to zero and then $a_{\mu \mu} \in \sigma(A)$.

In the following proposition extending the result in Remark 3(ii), the spectral radius of a special nonnegative matrix is formulated, whose all off-diagonal elements of the row with the maximum row sum are equal to zero.

Proposition 4. Let $A \in M_{n}(\mathbb{R})$ be a nonnegative matrix with $r_{\mu}(A)$ in (3) and $r_{\mu}(A)=a_{\mu \mu}$. Then

$$
\rho(A)=a_{\mu \mu} \text {. }
$$

Proof. Consider $r_{\mu}(A)=a_{\mu \mu}$; due to the definition of $r_{\mu}(A)$ in Lemma 1 and for all $1 \leq i \leq n$, we can write $a_{\mu \mu}=r_{\mu}(A) \geq r_{i}(A) \geq a_{i i}$, which implies $a_{\mu \mu}=\max _{1 \leq i \leq n}\left\{a_{i i}\right\}$. Combining the latter equality with the inequality (2) in Lemma 1 we derive

$a_{\mu \mu}=\max _{1 \leq i \leq n}\left\{a_{i i}\right\} \leq \rho(A)$.

Moreover, the inequality on the right side in (3) is written $\rho(A) \leq r_{\mu}(A)=a_{\mu \mu}$.

Hence, the validity in (6) follows from (7) and (8).

In the following proposition a lower bound of the above quantities $Z_{i}$ in (4) is formulated and upper and lower bounds for $w_{j}$ in (5) are provided.

Proposition 5. Let $A \in M_{n}(\mathbb{R})$ be a nonnegative matrix with $r_{\lambda}(A), r_{\mu}(A)$ in (3) and for $1 \leq i, j \leq n$ and $i \neq \lambda$, $j \neq \mu, z_{i}, w_{j}$ as in (4), (5), respectively. Let

$$
\begin{gathered}
z=\min \left\{z_{i}: 1 \leq i \leq n, \quad \text { with } i \neq \lambda\right\} \\
w=\max \left\{w_{j}: 1 \leq j \leq n, \quad \text { with } j \neq \mu\right\}
\end{gathered}
$$

Then,

$$
\begin{aligned}
z_{i} & \geq 1 \text { and } z \geq 1, \\
0 \leq w_{j} & \leq 1 \text { and } 0 \leq w \leq 1
\end{aligned}
$$

Proof. Consider $r_{\lambda}(A)>a_{\lambda \lambda}$. Due to the definition of $r_{\lambda}(A)$ in (3) and $z_{i}$ in (4a) with $b_{i}=a_{i \lambda}+a_{\lambda \lambda}-r_{i}(A)$, for $1 \leq i \leq n$ and $i \neq \lambda$, we can write the following inequalities:

$$
\begin{aligned}
& r_{i}(A) \geq r_{\lambda}(A) \Leftrightarrow \\
& a_{\lambda \lambda}+a_{i \lambda}-b_{i} \geq r_{\lambda}(A) \stackrel{\substack{b_{i}=a_{i \lambda}+a_{\lambda \lambda}-r_{i}(A) \\
a_{i \lambda}}}{\Leftrightarrow} r_{\lambda}(A)-a_{\lambda \lambda}+b_{i} \Leftrightarrow \\
& 4 a_{i \lambda}\left(r_{\lambda}(A)-a_{\lambda \lambda}\right) \geq 4\left(r_{\lambda}(A)-a_{\lambda \lambda}\right)\left(r_{\lambda}(A)-a_{\lambda \lambda}+b_{i}\right) \Leftrightarrow \\
& b_{i}^{2}+4 a_{i \lambda}\left(r_{\lambda}(A)-a_{\lambda \lambda}\right) \geq b_{i}^{2}+4\left(r_{\lambda}(A)-a_{\lambda \lambda}\right)\left(r_{\lambda}(A)-a_{\lambda \lambda}+b_{i}\right) \Leftrightarrow \\
& b_{i}^{2}+4 a_{i \lambda}\left(r_{\lambda}(A)-a_{\lambda \lambda}\right) \geq\left(b_{i}+2\left(r_{\lambda}(A)-a_{\lambda \lambda}\right)\right)^{2} \Leftrightarrow \\
& \sqrt{b_{i}^{2}+4 a_{i \lambda}\left(r_{\lambda}(A)-a_{\lambda \lambda}\right)} \\
& \quad \geq\left|b_{i}+2\left(r_{\lambda}(A)-a_{\lambda \lambda}\right)\right| \geq b_{i}+2\left(r_{\lambda}(A)-a_{\lambda \lambda}\right) \Leftrightarrow
\end{aligned}
$$




$$
\begin{aligned}
& -b_{i}+\sqrt{b_{i}^{2}+4 a_{i \lambda}\left(r_{\lambda}(A)-a_{\lambda \lambda}\right)} \geq 2\left(r_{\lambda}(A)-a_{\lambda \lambda}\right) \Leftrightarrow \\
& \frac{-b_{i}+\sqrt{b_{i}^{2}+4 a_{i \lambda}\left(r_{\lambda}(A)-a_{\lambda \lambda}\right)}}{2\left(r_{\lambda}(A)-a_{\lambda \lambda}\right)} \geq 1 \Leftrightarrow z_{i} \geq 1
\end{aligned}
$$

Thus, when $r_{\lambda}(A)>a_{\lambda \lambda}, z_{i} \geq 1$, for $1 \leq i \leq n$, and $i \neq \lambda$.

Consider $r_{\lambda}(A)=a_{\lambda \lambda}$; it is obvious that $z_{i}=i \geq 1$, due to $1 \leq i \leq n$.

Hence, the validity of (11) follows from the latter inequalities of $z_{i}$ for all $1 \leq i \leq n$, and $i \neq \lambda$ and the definition of $z$ in (9).

Consider $r_{\mu}(A)>a_{\mu \mu}$ and the definition of $w_{j}$ in $(5 a)$ with

$c_{j}=a_{j \mu}+a_{\mu \mu}-r_{j}(A)$, for $1 \leq j \leq n, j \neq \mu$. Since

$\sqrt{c_{j}^{2}+4 a_{j \mu}\left(r_{\mu}(A)-a_{\mu \mu}\right)} \geq\left|c_{j}\right| \geq c_{j} \Leftrightarrow$

$-c_{j}+\sqrt{c_{j}^{2}+4 a_{j \mu}\left(r_{\mu}(A)-a_{\mu \mu}\right)} \geq 0$,

and $r_{\mu}(A)-a_{\mu \mu}>0$, the quantity $w_{j}$ in (5a) yields

$w_{j} \geq 0$, for $1 \leq i \leq n, i \neq \mu$.

Moreover, due to the definition of $r_{\mu}(A)$ in Lemma 1 and the nonnegativity of the quantity

$$
\begin{array}{r}
c_{j}+2\left(r_{\mu}(A)-a_{\mu \mu}\right)=a_{j \mu}+a_{\mu \mu}-r_{j}(A)+2 r_{\mu}(A)-2 a_{\mu \mu} \\
=\left(r_{\mu}(A)-r_{j}(A)\right)+\left(r_{\mu}(A)-a_{\mu \mu}\right)+a_{j \mu}>0
\end{array}
$$

we can write the following:

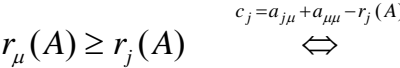

$$
\begin{aligned}
& r_{\mu}(A) \geq a_{j \mu}+a_{\mu \mu}-c_{j} \Leftrightarrow \\
& r_{\mu}(A)-a_{\mu \mu}+c_{j} \geq a_{j \mu} \geq 0 \quad \stackrel{r_{\mu}(A)>a_{\mu \mu}}{\Leftrightarrow} \\
& 4\left(r_{\mu}(A)-a_{\mu \mu}\right)\left(r_{\mu}(A)-a_{\mu \mu}+c_{j}\right) \geq 4 a_{j \mu}\left(r_{\mu}(A)-a_{\mu \mu}\right) \Leftrightarrow \\
& c_{j}^{2}+4\left(r_{\mu}(A)-a_{\mu \mu}\right)^{2}+4 c_{j}\left(r_{\mu}(A)-a_{\mu \mu}\right) \geq c_{j}^{2}+4 a_{j \mu}\left(r_{\mu}(A)-a_{\mu \mu}\right) \Leftrightarrow \\
& \left(c_{j}+2\left(r_{\mu}(A)-a_{\mu \mu}\right)\right)^{2} \geq c_{j}^{2}+4 a_{j \mu}\left(r_{\mu}(A)-a_{\mu \mu}\right)>0 \Leftrightarrow \\
& \sqrt{\left(c_{j}+2\left(r_{\mu}(A)-a_{\mu \mu}\right)\right)^{2}} \geq \sqrt{c_{j}^{2}+4 a_{j \mu}\left(r_{\mu}(A)-a_{\mu \mu}\right)} \Leftrightarrow \\
& \left|c_{j}+2\left(r_{\mu}(A)-a_{\mu \mu}\right)\right| \geq \sqrt{c_{j}^{2}+4 a_{j \mu}\left(r_{\mu}(A)-a_{\mu \mu}\right)} \quad \stackrel{(15)}{\Leftrightarrow} \\
& c_{j}+2\left(r_{\mu}(A)-a_{\mu \mu}\right) \geq \sqrt{c_{j}^{2}+4 a_{j \mu}\left(r_{\mu}(A)-a_{\mu \mu}\right)} \Leftrightarrow \\
& -c_{j}+\sqrt{c_{j}^{2}+4 a_{j \mu}\left(r_{\mu}(A)-a_{\mu \mu}\right)} \leq 2\left(r_{\mu}(A)-a_{\mu \mu}\right) \Leftrightarrow \\
& \frac{-c_{j}+\sqrt{c_{j}^{2}+4 a_{j \mu}\left(r_{\mu}(A)-a_{\mu \mu}\right)}}{2\left(r_{\mu}(A)-a_{\mu \mu}\right)} \leq 1 \Leftrightarrow w_{j} \leq 1
\end{aligned}
$$

Consider the other case, $r_{\mu}(A)=a_{\mu \mu}$; due to the definition of $w_{j}=1 / j$ with $1 \leq j \leq n$ in $(5 \mathrm{~b})$, it is obvious that $0<w_{j} \leq 1$.

Hence, the validity of (12) follows from (14), (16) and the latter inequalities $0<w_{j} \leq 1$, for all $1 \leq j \leq n, j \neq \mu$, as well as the definition of $w$ in (10).

Remark 6. Notice that :

(i) For $1 \leq j \leq n, j \neq \mu$, the following equivalence is derived by $(5 \alpha)$ :

$$
\begin{aligned}
& w_{j}=0 \Leftrightarrow a_{j \mu}=0 \text { and } c_{j} \geq 0 \\
& w_{j}=0 \Leftrightarrow a_{j \mu}=0 \text { and } r_{j}(A) \leq a_{\mu \mu}
\end{aligned}
$$

Combining the definition in (10) and the latter equivalence give the following

$w=0 \Leftrightarrow$

$$
a_{j \mu}=0 \text { and } r_{j}(A) \leq a_{\mu \mu}, \text { for all } j=1, \ldots, n, j \neq \mu
$$

(ii) The equality $z_{i}=1$ holds in (11) if and only if there exist at least two rows with the minimum row sum, which is proved by the equivalence in (13).

In this case, $z=1$ due to the definition in (9).

(iii) The equality $w_{j}=1$ holds in (12) if and only if there exist at least two rows with the maximum row sum, which is proved by the equivalence in (16).

In this case, $w=1$ due to the definition in (10).

In the following theorem, a new lower bound of the spectral radius of $A \geq 0$ is formulated, which can be compared by the Frobenius' lower bound in (3).

Theorem 7. Let $A \in M_{n}(\mathbb{R})$ be a nonnegative matrix with $r_{\lambda}(A)$ in (3) and $z$ in (9). Then,

$$
\rho(A) \geq z\left(r_{\lambda}(A)-a_{\lambda \lambda}\right)+a_{\lambda \lambda} .
$$

Proof. For $1 \leq i \leq n$ and $i \neq \lambda$, consider the quantities $z_{i}$ in (4a)-(4b) and the minimum of $z_{i}$ denote $z_{t}$, i.e., $z \equiv z_{t}=\min \left\{z_{i}: 1 \leq i \leq n\right.$, with $\left.i \neq \lambda\right\}$.

Consider the $n \times n$ nonsingular matrix $U_{z}=\operatorname{diag}(1, \ldots, 1,1 / z, 1, \ldots, 1)$, where its $\lambda$ diagonal element is equal to $1 / \mathrm{z}$ and let

$$
B_{z}=U_{z}^{-1} A U_{z}=\left(\begin{array}{ccccc}
a_{11} & \cdots & \frac{a_{1 \lambda}}{z} & \cdots & a_{1 n} \\
\vdots & & \vdots & & \vdots \\
z a_{\lambda 1} & \cdots & a_{\lambda \lambda} & \cdots & z a_{\lambda n} \\
\vdots & & \vdots & & \vdots \\
a_{n 1} & \cdots & \frac{a_{n \lambda}}{z} & \cdots & a_{n n}
\end{array}\right)
$$

Here, notice that when all the formulas of $z_{i}$ are given by (4b), then $z=z_{t}=1$ due to (9) and $B_{z} \equiv A$ in (19); in this case the inequality (18) follows immediately from the left side of (3) in Lemma 1.

Now, the $\lambda$-row sum of $B_{z}$ in (19) is given by

$r_{\lambda}\left(B_{z}\right)=\sum_{\substack{i=1 \\ i \neq \lambda}}^{n} z a_{\lambda i}+a_{\lambda \lambda}=z\left(r_{\lambda}(A)-a_{\lambda \lambda}\right)+a_{\lambda \lambda}$

and the $i$-row sum of $B_{z}$ for $1 \leq i \leq n$ with $i \neq \lambda$

$r_{i}\left(B_{z}\right)=r_{i}(A)-a_{i \lambda}+\frac{a_{i \lambda}}{z}$.

We claim that for $i=t$ holds

$r_{\lambda}\left(B_{z}\right)=r_{t}\left(B_{z}\right)$

and for $1 \leq i \leq n$ and $i \neq \lambda, t$ holds

$r_{i}\left(B_{z}\right) \geq r_{\lambda}\left(B_{z}\right)$. 
For $i=t$, since the formula of $z_{t}$ are given by (4a),

$z \equiv z_{t}=\frac{-b_{t}+\sqrt{b_{t}^{2}+4 a_{t \lambda}\left(r_{\lambda}(A)-a_{\lambda \lambda}\right)}}{2\left(r_{\lambda}(A)-a_{\lambda \lambda}\right)}$, with

$b_{t}=a_{t \lambda}+a_{\lambda \lambda}-r_{t}(A)$; substituting $z$ into (20) arises

$r_{\lambda}\left(B_{z}\right)=\frac{-b_{t}+\sqrt{b_{t}^{2}+4 a_{t \lambda}\left(r_{\lambda}(A)-a_{\lambda \lambda}\right)}}{2}+a_{\lambda \lambda}$.

Moreover, substituting the quantity $z$ into (21) for $i=t$ and using the equality $r_{t}(A)-a_{t \lambda}=a_{\lambda \lambda}-b_{t}$ arises

$r_{t}\left(B_{z}\right)=\frac{-b_{t}+\sqrt{b_{t}^{2}+4 a_{t \lambda}\left(r_{\lambda}(A)-a_{\lambda \lambda}\right)}}{2}+a_{\lambda \lambda}$.

Obviously, the first claim in (22) is proved by (24), (25).

Furthermore, due to the choice of $z$ in (9) it is obvious that $z \leq z_{i}$, for $1 \leq i \leq n$ and $i \neq \lambda, t$. Thus, using (4a), (20), (21) and the latter inequality $z \leq z_{i}$, we can write

$$
\begin{gathered}
r_{i}\left(B_{z}\right)-r_{\lambda}\left(B_{z}\right)=r_{i}(A)-a_{i \lambda}+\frac{a_{i \lambda}}{z}-z\left(r_{\lambda}(A)-a_{\lambda \lambda}\right)-a_{\lambda \lambda} \\
\geq r_{i}(A)-a_{i \lambda}-a_{\lambda \lambda}+\frac{a_{i \lambda}}{z}-z_{i}\left(r_{\lambda}(A)-a_{\lambda \lambda}\right) \\
\geq-b_{i}-z_{i}\left(r_{\lambda}(A)-a_{\lambda \lambda}\right)+\frac{a_{i \lambda}}{z_{i}} \\
=-b_{i}-\frac{-b_{i}+\sqrt{b_{i}^{2}+4 a_{i \lambda}\left(r_{\lambda}(A)-a_{\lambda \lambda}\right)}}{2\left(r_{\lambda}(A)-a_{\lambda \lambda}\right)}\left(r_{\lambda}(A)-a_{\lambda \lambda}\right) \\
+\frac{2 a_{i \lambda}\left(r_{\lambda}(A)-a_{\lambda \lambda}\right)}{-b_{i}+\sqrt{b_{i}^{2}+4 a_{i \lambda}\left(r_{\lambda}(A)-a_{\lambda \lambda}\right)}}=0,
\end{gathered}
$$

which proves the second claim in (23).

Now, combining (22), (23) and (20) arises

$\min \left\{r_{i}\left(B_{z}\right): 1 \leq i \leq n\right\}=r_{\lambda}\left(B_{z}\right)=z\left(r_{\lambda}(A)-a_{\lambda \lambda}\right)+a_{\lambda \lambda}$.

Moreover, the similarity of the matrices $A$ and $B_{z}$ by (19), the inequality (3) in Lemma 1 and (26) arise us write

$$
\rho(A)=\rho\left(B_{z}\right) \geq \min _{1 \leq i \leq n}\left\{r_{i}\left(B_{z}\right)\right\}=z\left(r_{\lambda}(A)-a_{\lambda \lambda}\right)+a_{\lambda \lambda},
$$

which completes the proof.

\section{Remark 8.}

Since $z \geq 1$ by (11), the proposed lower bound of Theorem 7 compared by the Frobenius' lower bound in (3)

$z\left(r_{\lambda}(A)-a_{\lambda \lambda}\right)+a_{\lambda \lambda} \geq r_{\lambda}(A)-a_{\lambda \lambda}+a_{\lambda \lambda}=r_{\lambda}(A)$,

thus the inequality in (18) is written

$r_{\lambda}(A) \leq z\left(r_{\lambda}(A)-a_{\lambda \lambda}\right)+a_{\lambda \lambda} \leq \rho(A)$.

i) Since the inequality $r_{\lambda}(A)<z\left(r_{\lambda}(A)-a_{\lambda \lambda}\right)+a_{\lambda \lambda}$ yields

$\left(r_{\lambda}(A)-a_{\lambda \lambda}\right)(1-z)<0$

and $z \geq 1$, the latter inequality follows that the new lower bound of Theorem 7 is sharper than the Frobenius' lower bound in (3) if and only if

$$
r_{\lambda}(A)>a_{\lambda \lambda} \text { and } z>1 \text {. }
$$

ii) It is obvious from (i) that the new lower bound of the spectral radius of $A \geq 0$ is equal to the Frobenius' one, when holds $r_{\lambda}(A)=a_{\lambda \lambda}$ or $z=1$.

According to Remarks 3(i) and 6(ii) the latter condition implies that the new lower bound is equal to the Frobenius' bound either all off-diagonal elements of the $\lambda$-th row are equal to zero or there are at least two rows with the minimal row sum.

In the following theorem, a new upper bound of the spectral radius is formulated, which can be compared by the Frobenius' upper bound in (3).

Theorem 9. Let $A \in M_{n}(\mathbb{R})$ be a nonnegative matrix with $r_{\mu}(A)$ in (3). Let the quantity $w$ in (10) with $w>0$. Then,

$$
\rho(A) \leq w\left(r_{\mu}(A)-a_{\mu \mu}\right)+a_{\mu \mu} .
$$

Proof. For $1 \leq j \leq n$ and $j \neq \mu$, consider the quantities $w_{j}$ in $(5 a)-(5 b)$ and the maximum of $w_{j}$ denote $w_{k}$, i.e., $w \equiv w_{k}=\max \left\{w_{j}: 1 \leq j \leq n\right.$, with $\left.j \neq \mu\right\}$.

Consider the $n \times n$ nonsingular matrix $V_{w}=\operatorname{diag}(1, \ldots, 1,1 / w, 1, \ldots, 1)$, where its $\mu$ diagonal element is equal to $1 / w$ and let

$$
C_{w}=V_{w}^{-1} A V_{w}=\left(\begin{array}{ccccc}
a_{11} & \cdots & \frac{a_{1 \mu}}{w} & \cdots & a_{1 n} \\
\vdots & & \vdots & & \vdots \\
w a_{\mu 1} & \cdots & a_{\mu \mu} & \cdots & w a_{\mu n} \\
\vdots & & \vdots & & \vdots \\
a_{n 1} & \cdots & \frac{a_{n \mu}}{w} & \cdots & a_{n n}
\end{array}\right)
$$

Here, notice that when all the formulas of $w_{j}$ are given by (5b), then $w=w_{k}=1$ due to (10) and $C_{w} \equiv A$ in (30); in this case the inequality (29) follows immediately from the right side of (3) in Lemma 1.

Now, the $\mu$-row sum of $C_{w}$ in (30) is given by

$$
r_{\mu}\left(C_{w}\right)=\sum_{\substack{j=1 \\ j \neq \mu}}^{n} w a_{\mu j}+a_{\mu \mu}=w\left(r_{\mu}(A)-a_{\mu \mu}\right)+a_{\mu \mu}
$$

and the $j$-row sum of $C_{w}$ for $j \neq \mu$ is given by

$r_{j}\left(C_{w}\right)=r_{j}(A)-a_{j \mu}+\frac{a_{j \mu}}{w}$.

We claim that for $j=k$ holds

$r_{\mu}\left(C_{w}\right)=r_{k}\left(C_{w}\right)$

and for $1 \leq j \leq n$ and $j \neq \mu, k$ holds

$r_{\mu}\left(C_{w}\right) \geq r_{j}\left(C_{w}\right)$.

For $j=k$, since the formula of $w_{k}$ is given by $(5 a)$,

$w \equiv w_{k}=\frac{-c_{k}+\sqrt{c_{k}^{2}+4 a_{k \mu}\left(r_{\mu}(A)-a_{\mu \mu}\right)}}{2\left(r_{\mu}(A)-a_{\mu \mu}\right)}$, with

$c_{k}=a_{k \mu}+a_{\mu \mu}-r_{k}(A)$, substituting $w$ into (31) arises

$r_{\mu}\left(C_{w}\right)=\frac{-c_{k}+\sqrt{c_{k}^{2}+4 a_{k \mu}\left(r_{\mu}(A)-a_{\mu \mu}\right)}}{2}+a_{\mu \mu}$.

Moreover, substituting the quantity $w$ into (32) for $j=k$ and using the equality $r_{k}(A)-a_{k \mu}=a_{\mu \mu}-c_{k}$ arises

$r_{k}\left(C_{w}\right)=\frac{-c_{k}+\sqrt{c_{k}^{2}+4 a_{k \mu}\left(r_{\mu}(A)-a_{\mu \mu}\right)}}{2}+a_{\mu \mu}$. 
Obviously, from (35), (36) the first claim in (33) is proved. Furthermore, due to the choice of $w$ in (10) it is obvious that $w \geq w_{j}$, for $1 \leq j \leq n$ and $j \neq \mu, k$. Thus, using (5a), (31), (32) and the latter inequality $w \geq w_{j}$, we can write

$$
\begin{gathered}
r_{j}\left(C_{w}\right)-r_{\mu}\left(C_{w}\right)=r_{j}(A)-a_{j \mu}+\frac{a_{j \mu}}{w}-w\left(r_{\mu}(A)-a_{\mu \mu}\right)-a_{\mu \mu} \\
\leq r_{j}(A)-a_{j \mu}-a_{\mu \mu}+\frac{a_{j \mu}}{w_{j}}-w_{j}\left(r_{\mu}(A)-a_{\mu \mu}\right) \\
=-c_{j}-w_{j}\left(r_{\mu}(A)-a_{\mu \mu}\right)+\frac{a_{j \mu}}{w_{j}} \\
=-c_{j}-\frac{-c_{j}+\sqrt{c_{j}^{2}+4 a_{j \mu}\left(r_{\mu}(A)-a_{\mu \mu}\right)}}{2\left(r_{\mu}(A)-a_{\mu \mu}\right)}\left(r_{\mu}(A)-a_{\mu \mu}\right) \\
+\frac{2 a_{j \mu}\left(r_{\mu}(A)-a_{\mu \mu}\right)}{-C_{j}+\sqrt{c_{j}^{2}+4 a_{j \mu}\left(r_{\mu}(A)-a_{\mu \mu}\right)}}=0,
\end{gathered}
$$

which proves the second claim in (34).

Now, combining (33), (34) and (31) arises

$\max _{1 \leq j \leq n}\left\{r_{j}\left(C_{w}\right)\right\}=r_{\mu}\left(C_{w}\right)=w\left(r_{\mu}(A)-a_{\mu \mu}\right)+a_{\mu \mu}$.

Moreover, the similarity of the matrices $A$ and $C_{w}$ by (30), the inequality (3) in Lemma 1 and (37) arise us write

$$
\rho(A)=\rho\left(C_{w}\right) \leq \max _{1 \leq j \leq n}\left\{r_{j}\left(C_{w}\right)\right\}=w\left(r_{\mu}(A)-a_{\mu \mu}\right)+a_{\mu \mu},
$$

which completes the proof.

\section{Remark 10.}

Since $0<w \leq 1$ by (12), the proposed upper bound of Theorem 9 compared by the Frobenius' upper bound in (3)

$w\left(r_{\mu}(A)-a_{\mu \mu}\right)+a_{\mu \mu} \leq r_{\mu}(A)-a_{\mu \mu}+a_{\mu \mu}=r_{\mu}(A)$,

thus the inequality in (29) is written

$\rho(A) \leq w\left(r_{\mu}(A)-a_{\mu \mu}\right)+a_{\mu \mu} \leq r_{\mu}(A)$.

i) Since the inequality $w\left(r_{\mu}(A)-a_{\mu \mu}\right)+a_{\mu \mu}<r_{\mu}(A)$ yields

$\left(r_{\mu}(A)-a_{\mu \mu}\right)(w-1)<0$

and $0<w \leq 1$ due to (12) and the assumption of Theorem 9, the latter inequality follows that the new upper bound of Theorem 9 is sharper than the Frobenius' upper bound in (3) if and only if

$$
r_{\mu}(A)>a_{\mu \mu} \text { and } 0<w<1 .
$$

ii) It is obvious from (i) that the new upper bound of the spectral radius of $A \geq 0$ is equal to the Frobenius' one, when holds $r_{\mu}(A)=a_{\mu \mu}$ or $w=1$.

According to Remark 3(iii) the case $r_{\mu}(A)=a_{\mu \mu}$ implies that the new upper bound is equal to the Frobenius' bound and due to Proposition 4 the spectral radius of $A$ is equal to $a_{\mu \mu}$.

According to Remark 6(iii) the case $w=1$ implies that the new upper bound is equal to the Frobenius' bound.

iii) The substitution $w=1 / u$ in the formula of the new upper bound of Theorem 9 gives the same upper bound of the spectral radius, which has been derived in [4, Theorem 3].
Example 11. Consider the matrix $A=\left(\begin{array}{llll}9 & 8 & 1 & 6 \\ 0 & 7 & 3 & 2 \\ 1 & 0 & 4 & 0 \\ 0 & 5 & 1 & 1\end{array}\right)$.

The spectrum of $A$ is $\sigma(A)=\{-0.4340,5.2412 \pm 1.7418 \mathrm{i}$, $10.9517\}$, which means that $\rho(A)=10.8213$. Clearly, its row sums are $r_{1}(A)=24, r_{2}(A)=12, r_{3}(A)=5, r_{4}(A)=7$, whereby $r_{\lambda}(A)=5, r_{\mu}(A)=24$, and $\lambda=3, \mu=1$.

Since $r_{3}(A)>a_{33}$, the quantities $z_{i}, i=1,2,4$ are given by $(4 \alpha)$ and these are equal to $z_{1}=\frac{19+\sqrt{365}}{2}, z_{2}=\frac{5+\sqrt{37}}{2}$, and $z_{4}=\frac{2+\sqrt{8}}{2}=1+\sqrt{2}$.

By (9), $z \equiv z_{4}=\min \left\{z_{1}, z_{2}, z_{4}\right\}=1+\sqrt{2}$.

Hence, the inequality in (18) of Theorem 7 gives $\rho(A) \geq(1+\sqrt{2})(5-4)+4=5+\sqrt{2}=6.4142$.

Since $r_{3}(A)>a_{33}$ and $z=1+\sqrt{2}>1$, it is obvious that the condition in (28) is verified, thus the proposed lower bound of $\rho(A)$ is sharper than the Frobenius' bound in (3). Here, notice that the largest diagonal element of $A$ is $a_{11}=9$, thus the Frobenius' bound in (2) is sharper than the proposed lower bound; the explanation is given in Proposition 17(ii) and Remark 18.

Since $r_{1}(A)>a_{11}$, the quantities $w_{j}, j=2,3,4$ are given by (5a) and these are equal to $w_{2}=\frac{1}{5}, w_{3}=\frac{-5+\sqrt{85}}{30}$, and $w_{4}=0$, since $c_{4}=a_{41}+a_{11}-r_{4}(A)=2>0$ and $a_{41}=0$. By (10), $w=\max \left\{w_{2}, w_{3}, w_{4}\right\}=1 / 5$.

Hence, the inequality in (29) of Theorem 9 gives $\rho(A) \leq \frac{1}{5}(24-9)+9=12$.

Since $r_{1}(A)>a_{11}$ and $w=1 / 5<1$, it is obvious that the condition in (39) is verified, thus the proposed upper bound of $\rho(A)$ is sharper than the Frobenius' bound in (3).

In the following proposition, the exact computation of the spectral radius of a pecial nonnegative matrix is achieved, using the new upper and lower bounds in Theorems 8,9. The spectral radius depends on the maximum and minimum row sum of $A$ and the elements of the first column of $A$.

Proposition 12. Let $A \in M_{n}(\mathbb{R})$ be a nonnegative with $a_{i 1}=a>0$, for $2 \leq i \leq n$, and row sums $r_{i}(A)$ such that $r_{1}(A)>r_{2}(A)=\cdots=r_{n}(A) \equiv r$, and $r_{1}(A)>a_{11}$. Then,

$\rho(A)=\frac{r-a+a_{11}+\sqrt{\left(a+a_{11}-r\right)^{2}+4 a\left(r_{1}(A)-a_{11}\right)}}{2}$.

Proof. Since $a_{i 1}=a>0$, for $2 \leq i \leq n$, and

$$
r_{1}(A)>r_{2}(A)=\cdots=r_{n}(A)=r>0,
$$

it is obvious that the quantities $w_{i}$ are given by $(5 a)$ for $c \equiv c_{2}=c_{3}=\cdots=c_{n}=a+a_{11}-r$, and due to (10) arises 


$$
\begin{aligned}
w \equiv w_{2} & =\cdots=w_{n}=\frac{-c+\sqrt{c^{2}+4 a\left(r_{1}(A)-a_{11}\right)}}{2\left(r_{1}(A)-a_{11}\right)} \\
& =\frac{r-a-a_{11}+\sqrt{\left(a+a_{11}-r\right)^{2}+4 a\left(r_{1}(A)-a_{11}\right)}}{2\left(r_{1}(A)-a_{11}\right)} .
\end{aligned}
$$

Notice that, $w>0$ because of the assumption $a_{i 1}=a>0$, for $2 \leq i \leq n$, and (17).

Consider the $n \times n$ nonsingular matrix $V_{w}=\operatorname{diag}\left(\frac{1}{w}, 1, \ldots, 1\right)$ and the corresponding matrix $C_{w}$ in (30), which is given by

$$
C_{w}=V_{w}^{-1} A V_{w}=\left(\begin{array}{cccc}
a_{11} & w a_{12} & \cdots & w a_{1 n} \\
\frac{a}{w} & a_{22} & \cdots & a_{2 n} \\
\vdots & \vdots & & \vdots \\
\frac{a}{w} & a_{n 2} & \cdots & a_{n n}
\end{array}\right)
$$

Substituting the quantity $w$ from (41) into the 1-row sum of $C_{w}$ in (42) arises

$$
\begin{aligned}
r_{1}\left(C_{w}\right) & =w\left(r_{1}(A)-a_{11}\right)+a_{11} \\
& =\frac{r-a+a_{11}+\sqrt{\left(a+a_{11}-r\right)^{2}+4 a\left(r_{1}(A)-a_{11}\right)}}{2} .
\end{aligned}
$$

Moreover, the substitution of the quantity $w$ from (41) in the formula of the $i$-row sum of $C_{w}$ gives

$$
\begin{aligned}
r_{2}\left(C_{w}\right) & =\cdots=r_{n}\left(C_{w}\right)=r-a+\frac{a}{w} \\
& =r-a+\frac{2 a\left(r_{1}(A)-a_{11}\right)}{r-a-a_{11}+\sqrt{\left(a+a_{11}-r\right)^{2}+4 a\left(r_{1}(A)-a_{11}\right)}} \\
& =\frac{r-a+a_{11}+\sqrt{\left(a+a_{11}-r\right)^{2}+4 a\left(r_{1}(A)-a_{11}\right)}}{2} .
\end{aligned}
$$

Obviously, since the second parts of the equalities in (43), (44) are identified, for the row sums of $C_{w}$ holds $r_{1}\left(C_{w}\right)=r_{2}\left(C_{w}\right)=\cdots=r_{n}\left(C_{w}\right)$.

Combining (45) with (3) of Lemma 1 we derive

$$
\begin{aligned}
\rho\left(C_{w}\right) & =\min _{1 \leq i \leq n}\left\{r_{i}\left(C_{w}\right)\right\} \equiv \max _{1 \leq i \leq n}\left\{r_{i}\left(C_{w}\right)\right\}=r_{2}\left(C_{w}\right) \\
& \stackrel{\text { (42) }}{=} \frac{r-a+a_{11}+\sqrt{\left(a+a_{11}-r\right)^{2}+4 a\left(r_{1}(A)-a_{11}\right)}}{2},
\end{aligned}
$$

which completes the proof of (40) due to the similarity of the matrices $A$ and $C_{w}$ in (42).

In the following proposition, the spectral radius of the special nonnegative matrix, whose elements are given under the restrictions in Remark 6(i) be indicated, is formulated.

Proposition 13. Let $A \in M_{n}(\mathbb{R})$ be a n onnegative matrix with $r_{\mu}(A)$ in $(3)$ and $w=0$, then

$$
\rho(A)=a_{\mu \mu} .
$$

Proof. Consider $w=0$; due to the equivalence in (17) the nonnegative matrix $A$ is formulated

$$
A=\left(\begin{array}{ccccc}
a_{11} & \cdots & 0 & \cdots & a_{1 n} \\
\vdots & & \vdots & & \vdots \\
a_{\mu 1} & \cdots & a_{\mu \mu} & \cdots & a_{\mu n} \\
\vdots & & \vdots & & \vdots \\
a_{n 1} & \cdots & 0 & \cdots & a_{n n}
\end{array}\right)
$$

with $r_{j}(A) \leq a_{\mu \mu}$, for all $j=1,2, \ldots, \mu-1, \mu+1, \ldots, n$, and its principal submatrix

$$
\tilde{A}=\left(\begin{array}{cccccc}
a_{11} & \cdots & a_{1, \mu-1} & a_{1, \mu+1} & \cdots & a_{1 n} \\
\vdots & & \vdots & \vdots & & \vdots \\
a_{\mu-1,1} & \cdots & a_{\mu-1, \mu-1} & a_{\mu-1, \mu+1} & \cdots & a_{\mu-1, n} \\
a_{\mu+1,1} & \cdots & a_{\mu+1, \mu-1} & a_{\mu+1, \mu+1} & \cdots & a_{\mu+1, n} \\
\vdots & & \vdots & \vdots & & \vdots \\
a_{n 1} & \cdots & a_{n, \mu-1} & a_{n, \mu+1} & \cdots & a_{n n}
\end{array}\right) .
$$

The formula of $\tilde{A}$ and (17) yield

$r_{j}(\tilde{A})=r_{j}(A) \leq a_{\mu \mu} \Rightarrow \max _{\substack{1 \leq j \leq n, j \neq \mu}}\left\{r_{j}(\tilde{A})\right\}=\max _{\substack{1 \leq j \leq n, j \neq \mu}}\left\{r_{j}(A)\right\} \leq a_{\mu \mu}$,

and by (3)

$$
\rho(\tilde{A}) \leq \max _{\substack{1 \leq j \leq n, j \neq \mu}}\left\{r_{j}(\tilde{A})\right\} \leq a_{\mu \mu} .
$$

Obviously, from the formula of $A$ arise $a_{\mu \mu} \in \sigma(A)$ and

$$
\sigma(A)=\left\{a_{\mu \mu}\right\} \cup \sigma(\tilde{A}) .
$$

Hence, the validity in (46) follows from the definition of the spectral radius of $A$ in (1), (47) and (48).

In the following proposition under the restriction concerning the minimum row sums of $\tilde{A}, A$ a new lower bound of $A \geq 0$ is formulated, which is derived by the lower bound of the spectral radius of $\tilde{A}$ and improves the Frobenius' one.

Proposition 14. Let $A \in M_{n}(\mathbb{R})$ be a n onnegative matrix with $r_{\lambda}(A)$ in (3). L et $\tilde{A}=\left(\tilde{a}_{i j}\right)_{i, j=1}^{n-1} \in M_{n-1}(\mathbb{R})$ be the principal submatrix obtained by deleting $\lambda$-row and $\lambda$ column of $A$. If $r_{\tilde{\lambda}}(\tilde{A})>r_{\lambda}(A)$, then

$$
r_{\lambda}(A)<\tilde{z}\left(r_{\tilde{\lambda}}(\tilde{A})-\tilde{a}_{\tilde{\lambda} \tilde{\lambda}}\right)+\tilde{a}_{\tilde{\lambda} \tilde{\lambda}} \leq \rho(A),
$$

where the associated quantities $r_{\tilde{\lambda}}(\tilde{A}), \tilde{a}_{\tilde{\lambda} \tilde{\lambda}}$ and $\tilde{z}$ are defined by (3) and (9) for $\tilde{A}$.

Proof. The relation between the spectral radii of principal submatrix $\tilde{A}$ of $A$ and the nonnegative matrix $A$ is given by

$$
\rho(\tilde{A}) \leq \rho(A),
$$

[11, Corollary, 8.1.20]. Applying Theorem 7 for the principal submatrix $\tilde{A}$ of $A$, the inequality (27) yields

$$
r_{\tilde{\lambda}}(\tilde{A}) \leq \tilde{z}\left(r_{\tilde{\lambda}}(\tilde{A})-\tilde{a}_{\tilde{\lambda} \tilde{\lambda}}\right)+\tilde{a}_{\tilde{\lambda} \tilde{\lambda}} \leq \rho(\tilde{A}) .
$$

Combining (50) with assumption $r_{\tilde{\lambda}}(\tilde{A})>r_{\lambda}(A)$ the latter inequality implies that

$r_{\lambda}(A)<r_{\tilde{\lambda}}(\tilde{A}) \leq \tilde{z}\left(r_{\tilde{\lambda}}(\tilde{A})-\tilde{a}_{\tilde{\lambda} \tilde{\lambda}}\right)+\tilde{a}_{\tilde{\lambda} \tilde{\lambda}} \leq \rho(\tilde{A}) \leq \rho(A)$,

which completes the proof. 
Remark 15. Remind that the new lower bound of the spectral radius of $A$ in (18) is sharper than the Frobenius' one under the conditions $r_{\lambda}(A)>a_{\lambda \lambda}$ and $z>1$ (see, Remark $8(i))$; here we are going to extend the above result in the cases $r_{\lambda}(A)=a_{\lambda \lambda}$ or $z=1$ using the result in Proposition 14 under the restriction $r_{\tilde{\lambda}}(\tilde{A})>r_{\lambda}(A)$ for the principal submatrix $\tilde{A}$ of $A \geq 0$. Obviously, the proposed lower bound $\tilde{z}\left(r_{\tilde{\lambda}}(\tilde{A})-\tilde{a}_{\tilde{\lambda} \tilde{\lambda}}\right)+\tilde{a}_{\tilde{\lambda} \tilde{\lambda}}$ in (49) of the spectral radius of $A$ is sharper than the Frobenius' one; the sharpness of the bound does not depend on the conditions in (28), it depends only on the condition concerning the minimum row sums of $\tilde{A}$ and $A$.

Example 16. Consider the matrix $A=\left(\begin{array}{llll}7 & 9 & 5 & 4 \\ 0 & 2 & 0 & 0 \\ 1 & 3 & 2 & 4 \\ 0 & 8 & 1 & 5\end{array}\right)$.

The spectrum of $A$ is $\sigma(A)=\{0.4831,2,5.2730,8.2439\}$, which means that $\rho(A)=8.2439$. Clearly, its row sums are $r_{1}(A)=25, r_{2}(A)=2, r_{3}(A)=10, r_{4}(A)=14$, whereby $r_{\lambda}(A)=2, r_{\mu}(A)=25$, and $\lambda=2, \mu=1$.

Since $r_{2}(A)=a_{22}=2$, the new lower bound of the spectral radius of $A$, which is established in Theorem 7 , is equal to the Frobenius' one (see, Remark 8(ii)). According to Remark 15 , consider the principal submatrix

$$
\tilde{A}=\left(\begin{array}{lll}
7 & 5 & 4 \\
1 & 2 & 4 \\
0 & 1 & 5
\end{array}\right),
$$

which is obtained by deleting the second row and column of A. Obviously, $\quad r_{\tilde{\mu}}(\tilde{A}) \equiv r_{1}(\tilde{A})=16 \quad, \quad r_{2}(\tilde{A})=7 \quad$ and $r_{\tilde{\lambda}}(\tilde{A}) \equiv r_{3}(\tilde{A})=6$. Since $r_{3}(\tilde{A})>\tilde{a}_{33}=5$, the quantities $\tilde{z}_{1}, \tilde{z}_{2}, \tilde{z}$ of $\tilde{A}$ are computed by (4a), (9), these are equal to $\tilde{z}_{1}=\frac{7+\sqrt{65}}{2}, \tilde{z}_{2}=-1+\sqrt{5}$ and $\tilde{z} \equiv \min \left\{\tilde{z}_{1}, \tilde{z}_{2}\right\}=-1+\sqrt{5}$. Since $r_{\tilde{\lambda}}(\tilde{A})>r_{\lambda}(A)$ the assumption of Proposition $14 \mathrm{i} \mathrm{s}$ satisfied, hence the new lower bound of $\rho(A)$ is formulated by (49) and equal to

$\tilde{z}\left(r_{\tilde{\lambda}}(\tilde{A})-\tilde{a}_{\tilde{\lambda} \tilde{\lambda}}\right)+\tilde{a}_{\tilde{\lambda} \tilde{\lambda}}=(-1+\sqrt{5})(6-5)+5=4+\sqrt{5}=6.2361$.

For the upper bound of $A$, since $r_{1}(A)>a_{11}$, the quantities $w_{j}, j=2,3,4$ are given by $(5 a)$ and these are equal to $w_{2}=0, w_{3}=\frac{1+\sqrt{19}}{18}, w_{4}=\frac{7}{18}$.

By (10), $w=\max \left\{w_{2}, w_{3}, w_{4}\right\}=7 / 18$. Hence, Theorem 9 can be applied and the inequality in (29) gives $\rho(A) \leq \frac{7}{18}(25-7)+7=14$.

Here, notice that the new bounds give: $6.2361 \leq \rho(A) \leq 14$ and the Frobenius' bounds: $2 \leq \rho(A) \leq 25$.

Obviously, the new lower bound of the spectral radius of $A$, which is computed through the new lower bound of the principal submatrix $\tilde{A}$ and formulated in (49), is sharper than the Frobenius' lower bound $r_{\lambda}(A) \equiv r_{2}(A)=2$.

Finally, in the following we are going to show how we are able to involve both the two Frobenius' lower bounds of Lemma 1 in the proposer lower bound determination of Theorem 7.

Remind that for a nonnegative matrix the determined Frobenius' lower bounds are: $a \equiv \max _{1 \leq i \leq n}\left\{a_{i i}\right\} \leq \rho(A)$ in (2) and $r_{\lambda}(A) \equiv \min _{1 \leq i \leq n}\left\{r_{i}(A)\right\} \leq \rho(A)$ in (3).

It is obvious that, if $a \leq r_{\lambda}(A)$ with $r_{\lambda}(A)>a_{\lambda \lambda}$ and $z>1$, then Theorem 7 determines a new lower bound, which is sharper than both the Frobenius' lower bounds due to Remark 8(i); in this case the new lower bound is formulated by (18) and denoted

$$
l b(z)=z\left(r_{\lambda}(A)-a_{\lambda \lambda}\right)+a_{\lambda \lambda}
$$

with $z=\min \left\{z_{i}: 1 \leq i \leq n\right.$, with $\left.i \neq \lambda\right\}$ in (9).

Furthermore, if $r_{\lambda}(A)<a$, then the new lower bound in (51) can be compared with both the Frobenius' lower bounds of Lemma 1 and the conditions defined to determine the sharper one.

The idea is to consider the lower bound a double-value function of $z, z_{a}$, where $z$ is denoted in (9) and $z_{a}$ is defined

$$
z_{a}=\frac{a-a_{\lambda \lambda}}{r_{\lambda}(A)-a_{\lambda \lambda}} .
$$

The corrected lower bound of $\rho(A)$ is denoted

$l b\left(z_{a}\right)=z_{a}\left(r_{\lambda}(A)-a_{\lambda \lambda}\right)+a_{\lambda \lambda}$.

Since the substitution of $z_{a}$ into the corrected lower bound of $\rho(A)$ yields $l b\left(z_{a}\right)=a$, it is obvious that we can write $l b\left(z_{a}\right)=a \leq \rho(A)$ due to Lemma 1 ; thus, $l b\left(z_{a}\right)$ consists of a lower bound of $\rho(A)$.

Now, the proposed lower bound in Theorem $7 \mathrm{c}$ an be compared with the associated Frobenius' lower bound of Lemma 1, as it is proved in the following proposition.

Proposition 17. Let $A \in M_{n}(\mathbb{R})$ be a nonnegative matrix and let $a \equiv \max _{1 \leq i \leq n}\left\{a_{i i}\right\}, r_{\lambda}(A), z, z_{a}$, and $l b(z)$ be defined by (2), (3), (9), (52) and (51), respectively.

i) If $a \leq r_{\lambda}(A)$ with $r_{\lambda}(A)>a_{\lambda \lambda}$ and $z \geq 1$, then $a \leq r_{\lambda}(A) \leq l b(z) \leq \rho(A)$.

ii) If $r_{\lambda}(A)<a$ with $r_{\lambda}(A)>a_{\lambda \lambda}$ and $z<z_{a}$, then $r_{\lambda}(A) \leq l b(z)<a \leq \rho(A)$.

iii) If $r_{\lambda}(A)<a$ with $r_{\lambda}(A)>a_{\lambda \lambda}$ and $z \geq z_{a}$, then $r_{\lambda}(A)<a \leq l b(z) \leq \rho(A)$.

Proof. i) The order of the lower bound of the spectral radius of $A$ follows immediately from Theorem 7 and the assumptions.

ii) Since $r_{\lambda}(A)-a_{\lambda \lambda}>0$, the inequality $z<z_{a}$ yields $z\left(r_{\lambda}(A)-a_{\lambda \lambda}\right)+a_{\lambda \lambda}<z_{a}\left(r_{\lambda}(A)-a_{\lambda \lambda}\right)+a_{\lambda \lambda}$.

By substituting $l b(z), z_{a}$ from (51) and (52), respectively, into the above inequality, we have $l b(z)<a$. 
The order of the bounds of the spectral radius of $A$ follows from the left inequality in (27) and (2).

iii) Since $r_{\lambda}(A)-a_{\lambda \lambda}>0$, the inequality $z \geq z_{a}$ yields $z\left(r_{\lambda}(A)-a_{\lambda \lambda}\right)+a_{\lambda \lambda} \geq z_{a}\left(r_{\lambda}(A)-a_{\lambda \lambda}\right)+a_{\lambda \lambda}$.

Following analogous statements as the proof in (ii) arises $l b(z) \geq a$.

The order of the bounds of the spectral radius of $A$ follows from the right inequality in (27) and the assumption $r_{\lambda}(A)<a$.

Remark 18. According to Proposition 17, the proposed lower bound $l b(z)$ in (51) of the spectral radius of $A$ is sharper than the both Frobenius' lower bounds of Lemma 1, when the assumptions in (i) or (iii) of Proposition 17 a re satisfied. Notice that the first assumption depends on the order of the minimum row sum $r_{\lambda}(A)$ and the maximum diagonal element $a$ of $A$ and the second depends on the quantity $z$.

Example 19. Consider the nonnegative matrices

$$
\begin{aligned}
A & =\left(\begin{array}{llll}
1 & 3 & 0 & 2 \\
2 & 4 & 5 & 3 \\
0 & 4 & 1 & 5 \\
4 & 1 & 5 & 2
\end{array}\right), B=\left(\begin{array}{llll}
9 & 7 & 5 & 4 \\
2 & 5 & 1 & 7 \\
1 & 3 & 2 & 4 \\
0 & 1 & 1 & 2
\end{array}\right) \text { and } \\
C & =\left(\begin{array}{llll}
9 & 6 & 5 & 0 \\
2 & 1 & 3 & 2 \\
4 & 7 & 8 & 3 \\
1 & 5 & 0 & 6
\end{array}\right) .
\end{aligned}
$$

- The spectrum of the nonnegative matrix $A$ is $\sigma(A)=\{-5.0566,1.0376 \pm 1.0754 \mathrm{i}, 10.9814\}$, which means that $\rho(A)=10.9814$.

Clearly, its row sums are $r_{\lambda}(A) \equiv r_{1}(A)=6$,

$r_{\mu}(A) \equiv r_{2}(A)=14, r_{3}(A)=10, r_{4}(A)=12$, and

$a \equiv \max _{1 \leq i \leq 4}\left\{a_{i i}\right\}=a_{22}=4$.

Since $a<r_{1}(A)$ and $r_{1}(A)>a_{11}$, the assumptions of Proposition 17(i) are satisfied, thus, the lower bounds of $\rho(A)$ are ordered as $4<r_{1}(A) \leq l b(z) \leq \rho(A)$ verifying the inequalities therein, where $l b(z)$ is formulated by (51).

The quantities $z_{i}, i=2,3,4$ are given by $(4 \alpha)$ and these are equal to $z_{2}=\frac{11+\sqrt{161}}{10}, z_{3}=\frac{9}{5}$, and $z_{4}=\frac{7+\sqrt{129}}{10}$. Since $Z \equiv z_{3}=\min \left\{z_{2}, z_{3}, z_{4}\right\}=9 / 5$, the lower bound in (51) is equal to $l b(z)=z\left(r_{1}(A)-a_{11}\right)+a_{11}=\frac{9}{5}(6-1)+1=10$, which is sharper than the both Frobenius' lower bounds in Lemma 1 due to Remark 18 .

Hence, $10 \leq \rho(A)$.

For the upper bound of $A$, since $r_{4}(A)>a_{44}$, the quantities $w_{j}, \quad j=1,2,3$ are given by ( $\left.5 a\right)$ and these are equal to $w_{1}=\frac{-1+\sqrt{121}}{20}=\frac{1}{2}, w_{3}=\frac{2+\sqrt{164}}{20}, w_{4}=\frac{7+\sqrt{89}}{20}$.

By (10), $w \equiv w_{4}=\max \left\{w_{1}, w_{3}, w_{4}\right\}=\frac{7+\sqrt{89}}{20}=0.8217$.

Hence, Theorem 9 can be applied and the inequality in (29) gives $\rho(A) \leq \frac{7+\sqrt{89}}{20}(14-4)+4=12.2170$.

Thus, the spectral radius is lower and upper bounded: $10 \leq \rho(A) \leq 12.217$

Here, notice that the both bounds (lower and upper) of $\rho(A)$ are given by the proposed bounds in Theorem 7 and 9 , respectively.

- The spectrum of $B$ is $\sigma(B)=\{0.3988 \pm 0.4882 \mathrm{i}$,

$4.5890,12.6135\}$, which means that $\rho(B)=12.6135$.

Clearly, its row sums are $r_{\mu}(B) \equiv r_{1}(B)=25, r_{2}(B)=15$, $r_{3}(B)=10, r_{\lambda}(B) \equiv r_{4}(B)=4$, and $a \equiv \max _{1 \leq i \leq 4}\left\{b_{i i}\right\}=b_{11}=9$.

Since $r_{4}(B)>b_{44}$, for $i=1,2,3$ the quantities $z_{i}$ are given by $(4 a)$ and these are equal to $Z_{1}=\frac{19+\sqrt{393}}{4}$, $z_{2}=\frac{6+\sqrt{92}}{4}$, and $z_{3}=\frac{4+\sqrt{48}}{4}=1+\sqrt{3}$.

By (9), $z \equiv z_{3}=\min \left\{z_{1}, z_{2}, z_{3}\right\}=1+\sqrt{3}=2.7321$.

Moreover, the quantities $z_{a}$ are computed by (52) and equal to $: z_{a}=\frac{a-b_{44}}{r_{4}(B)-b_{44}}=\frac{9-2}{4-2}=3.5$. In addition, by (51),

$l b(z)=z\left(r_{4}(B)-b_{44}\right)+b_{44}=(1+\sqrt{3})(4-2)+2=7.4641$.

Since $r_{4}(B)<a$ with $r_{4}(B)>b_{44}$ and $z<z_{a}$, the assumptions of Proposition 17(ii) are satisfied, thus, the lower bounds of $\rho(B)$ are ordered as

$r_{4}(B) \leq l b(z)<a \leq \rho(B)$, verifying the inequalities therein.

Hence, it is obvious that the Frobenius' lower bound in (2) is sharper than the Frobenius' lower bound in (3) and the proposed lower bound of Theorem 7 .

For the upper bound of $B$, since $r_{1}(B)>b_{11}$, the quantities $w_{j}, j=2,3,4$ are given by $(5 a)$ and these are equal to $w_{2}=\frac{4+\sqrt{144}}{32}=\frac{1}{2}, w_{3}=\frac{\sqrt{64}}{32}=\frac{1}{4}, w_{4}=0$.

By (10), $w \equiv w_{2}=\max \left\{w_{2}, w_{3}, w_{4}\right\}=1 / 2$. Hence, Theorem 9 can be applied and the inequality in (29) gives $\rho(B) \leq \frac{1}{2}(25-9)+9=17$.

Thus, the spectral radius is lower and upper bounded: $9 \leq \rho(B) \leq 17$

Here, notice that the lower bound of $\rho(B)$ is given by Frobenius' one in (2) and the upper bound of $\rho(B)$ by Theorem 9.

- The spectrum of $C$ is $\sigma(C)=\{-2.0292,4.6153,5.5443$, $15.8695\}$, which means that $\rho(C)=15.8695$. 
Clearly, its row sums are $r_{1}(C)=20, \quad r_{\lambda}(C) \equiv r_{2}(C)=8$, $r_{\mu}(C) \equiv r_{3}(C)=22, r_{4}(C)=12$ and $a \equiv \max _{1 \leq i \leq 4}\left\{c_{i i}\right\}=c_{11}=9$.

Since $r_{2}(C)>c_{22}=1$, for $i=1,3,4$ the quantities $z_{i}$ are given by $(4 a)$ and these are equal to $Z_{1}=\frac{13+\sqrt{337}}{14}$, $z_{3}=\frac{14+\sqrt{392}}{14}=\frac{7+\sqrt{98}}{7}$, and $z_{4}=\frac{6+\sqrt{176}}{14}=\frac{3+\sqrt{44}}{7}$.

By (9), $z \equiv z_{4}=\min \left\{z_{1}, z_{3}, z_{4}\right\}=\frac{3+\sqrt{44}}{7}=1.3762$.

Moreover, the quantities $z_{a}$ are computed by (52) and equal to :

$z_{a}=\frac{a-c_{22}}{r_{2}(C)-c_{22}}=\frac{9-1}{8-1}=\frac{8}{7}=1.1429$. In addition, by (51),

$l b(z)=z\left(r_{2}(C)-c_{22}\right)+c_{22}=\frac{3+\sqrt{44}}{7}(8-1)+1=10.6332$

Since $r_{2}(C)<a$ with $r_{2}(C)>c_{22}$ and $z>z_{a}$, the assumptions of Proposition 17(iii) are satisfied, thus, the lower bounds of $\rho(C)$ are ordered as

$r_{2}(C)<a \leq l b(z) \leq \rho(C)$, verifying the inequalities therein.

According to Remark 18, the proposed lower bound $l b(z)$ in (51) is sharper than the both Frobenius' lower bounds in Lemma 1 , thus $10.6332 \leq \rho(C)$.

For the upper bound of $C$, since $r_{3}(C)>c_{33}$, the quantities $w_{j}, j=1,2,4$ are given by $(5 a)$ and these are equal to $w_{1}=\frac{7+\sqrt{329}}{28}, w_{2}=\frac{-3+\sqrt{177}}{28}, w_{4}=\frac{8}{28}$.

By $(10), \quad w \equiv w_{1}=\max \left\{w_{1}, w_{2}, w_{4}\right\}=\frac{7+\sqrt{329}}{28}=0.8978$.

Hence, Theorem 9 can be applied and the inequality in (29)

gives $\rho(C) \leq \frac{7+\sqrt{329}}{28}(22-8)+8=20.5692$.

Thus, the spectral radius is lower and upper bounded:

$10.6332 \leq \rho(C) \leq 20.5692$

Here, notice that the both bounds (lower and upper) of $\rho(C)$ are given by the proposed bounds in Theorem 7 and 9 , respectively.

\section{APPLICATION IN STABILITY OF SISO LINEAR DISCRETE} TIME SYSTEMS

Consider the linear invariant discrete-time system with the following state space realization $[3,13,17]$

$x(k+1)=A x(k)+B u(k)$

$y(k)=C x(k), \quad$ for $k=0,1,2, \ldots$

where $x(k) \in \mathbb{R}^{n}, \quad u(k) \in \mathbb{R}^{p}, y(k) \in \mathbb{R}^{r}$ are the state, the control input and the measured output vectors, respectively. Moreover, $A \in M_{n}(\mathbb{R}), B \in M_{n \times p}(\mathbb{R})$ and $C \in M_{r \times n}(\mathbb{R})$ are given constant matrices. Such a system is often closed by the control law $u(k)=K y(k)$, which yields

$x(k+1)=(A+B K C) x(k)$, for $k=0,1,2, .$. where $K \in M_{p \times r}(\mathbb{R})$ is the output feedback gain matrix. The discrete-time control system in (54) is asymptotically stable, i.e. $\lim _{k \rightarrow \infty} x(k)=0$ for any initial vector $x(0)$, if and only if $\rho(A+B K C)<1$.

The eigenvalue assignment problem is to design an output feedback gain matrix $K \in M_{p \times r}(\mathbb{R})$ providing a closed-loop system in a satisfactory stage by shifting controllable eigenvalues to desirable locations in the complex plane. In particular, the solution of the problem requires the spectral radius of the closed-loop matrix $A+B K C$ to be strictly within the unit circle in the complex plane. Various approaches have been used to study the aspects of the stabilization problem and necessary conditions under which the problem can be stabilized have been proposed, but in some cases the problem is NP-hard [10]. The problem of the stabilization of the linear discrete-time system of the form in (53), where the vectors $x(k) \in \mathbb{R}^{n}, u(k) \in \mathbb{R}^{p}$ and the matrices $A \in M_{n}(\mathbb{R}), B \in M_{n \times p}(\mathbb{R})$ are nonnegative, has attracted the interest of many researches. Here, the discretetime control system in (53) is asymptotically stable, if and only if $\rho(A)<1,[13]$.

Thus the bounds of the spectral radius of $A$ can ensure the (asymptotically) stability of the system, which consists of the motivation of the following proposition. Using the new upper and lower bounds of Theorems 8,9, the results in Remarks 8(i), 11(i) and Proposition 4, a criterion is formulated, which depends on the quantities $z, w$, $r_{\mu}(A), r_{\lambda}(A), a_{\mu \mu}, a_{\lambda \lambda}$, in order to ensure the stability of a linear discrete-time system.

Proposition 20. Let $A \in M_{n}(\mathbb{R})$ be a n onnegative with $r_{\mu}(A)>a_{\mu \mu}$, and $r_{\lambda}(A)>\alpha_{\lambda \lambda}$. Then,

i) if $w<\frac{1-a_{\mu \mu}}{r_{\mu}(A)-a_{\mu \mu}}<1 \Rightarrow \rho(A)<1$.

ii) if $z>\frac{1-a_{\lambda \lambda}}{r_{\lambda}(A)-a_{\lambda \lambda}} \Rightarrow \rho(A)>1$.

Proof. i) Since $r_{\mu}(A)>a_{\mu \mu}$, we can write the following equivalence:

$$
\begin{aligned}
& w<\frac{1-a_{\mu \mu}}{r_{\mu}(A)-a_{\mu \mu}}<1 \Leftrightarrow \\
& w\left(r_{\mu}(A)-a_{\mu \mu}\right)<1-a_{\mu \mu}<r_{\mu}(A)-a_{\mu \mu} \Leftrightarrow \\
& w\left(r_{\mu}(A)-a_{\mu \mu}\right)+a_{\mu \mu}<1<r_{\mu}(A)
\end{aligned}
$$

The validity of (55) follows from the above equivalence and (38) in Remark 10.

ii) Since $r_{\lambda}(A)>a_{\lambda \lambda}$, we can write

$$
1<z\left(r_{\lambda}(A)-a_{\lambda \lambda}\right)+a_{\lambda \lambda} \Leftrightarrow z>\frac{1-a_{\lambda \lambda}}{r_{\lambda}(A)-a_{\lambda \lambda}} \text {. }
$$

The validity of (56) follows immediately from the above equivalence and (27) in Remark 8. 
Remark 21. Notice that Proposition 20 provides a criterion in order to investigate the property of stability of a discretetime control system in (53) except the computation of the spectral radius of the nonnegative matrix of the system and its compares with the unit. In particular, the system is asymptotically stable, when the assumption in (55) satisfies; the system is not asymptotically stable, when the assumption in (56) satisfies.

Example 22. Consider the single-input single-output (SISO) linear discrete-time system in [5, Example 8] with

$$
A=\left(\begin{array}{ccc}
0.5 & 0 & 0.6 \\
0.6 & 0.8 & 1.2 \\
0.8 & 1 & 0.8
\end{array}\right) \quad \text { and } B=\left(\begin{array}{ccc}
0.5 & 0 & 0.6 \\
0.0701 & 0.2799 & 0.5 \\
0.2701 & 0.4799 & 0.1
\end{array}\right)
$$

the associated closed-loop matrix.

- The spectrum of $A$ is $\sigma(A)=\{-0.4,0.3542,2.1458\}$, which means that $\rho(A)=2.1458$.

The row sums of $A$ are $r_{\lambda}(A) \equiv r_{1}(A)=1.1$, and $r_{\mu}(A) \equiv r_{2}(A)=r_{3}(A)=2.6$.

According to Remark 6 (iii), the equality $r_{2}(A)=r_{3}(A)=2.6$ follows $w=1$.

Since $r_{1}(A)>a_{11}$, the quantities $z_{i}, \quad i=2,3$ are given by $(4 a)$ and these are equal to $z_{2}=\frac{1.5+\sqrt{16.65}}{1.2}=4.6504$, and $Z_{3}=\frac{1.3+\sqrt{3.61}}{1.2}=2.6667$.

By (9), $z \equiv z_{3}=\min \left\{z_{2}, z_{3}\right\}$. Obviously, $z>0.5 / 0.6$. Since $r_{1}(A)>a_{11}$ and the assumption in (56) are satisfied, the associated linear discrete-time system is not asymptotically stable due to Remark 21.

Here, notice that the new bounds give: $2.1 \leq \rho(A) \leq 2.6$ and the Frobenius' bounds: $1.1 \leq \rho(A) \leq 2.6$.

Obviously, the new lower bound of the spectral radius of $A$, which is established in Theorem 7 and computed by (18), is sharper than the Frobenius'one.

- The spectrum of $B$ is $\sigma(B)=\{-0.4,0.3542,0.9257\}$, which means that $\rho(B)=0.9257$.

The row sums of the closed-loop matrix $B$ are $r_{\mu}(B) \equiv r_{1}(B)=1.1$, and $r_{\lambda}(B) \equiv r_{2}(B)=r_{3}(B)=0.85$.

According to Remark 6 (ii), the equality $r_{2}(B)=r_{3}(B)=0.85$ follows $z=1$.

Since $r_{1}(B)>b_{11}$, the quantities $w_{i}, i=2,3$ are given by (5a) and these are equal to

$w_{2}=\frac{0.2799+\sqrt{0.2466}}{1.2}=0.6471$,

and $w_{3}=\frac{0.0799+\sqrt{0.6546}}{1.2}=0.7408$.

By (10), $w \equiv w_{2}=\max \left\{w_{2}, w_{3}\right\}$. Obviously, $w<0.5 / 0.6<1$. Since $r_{\mu}(B)>b_{11}$ and the assumption in (55) are satisfied, the associated linear discrete-time system is asymptotically stable due to Remark 21.
Here, notice that the new bounds give: $0.85 \leq \rho(B) \leq 0.9445$ and the Frobenius' bounds:

$0.85 \leq \rho(B) \leq 1.1$.

Moreover, the new upper bound of the spectral radius of $B$, which is established in Theorem 9 and computed by (29), is sharper than the Frobenius' one. Note that the Frobenius' bounds of $\rho(B)$ cannot ensure, if the system is stable or not, while the new bounds ensure the stability of the system.

\section{ALGORITHMS AND COMPLEXITY}

In this section the algorithms for computing the upper and lower bounds of the spectral radius of a nonnegative matrix are summarized and their complexity is analyzed.

The algorithm for computing the lower bound of the spectral radius of a nonnegative matrix results from Lemma 1, Definition 2, Theorem 7 and Remark 8 and is summarized in the following.

\section{lower bound algorithm I}

input: nonnegative matrix $A$

output: upper bound $l b$ of the spectral radius of $A$

step 1. compute $r_{i}(A)=\sum_{j=1}^{n} a_{i j}, 1 \leq i \leq n$

step 2. find $r_{\lambda}(A)=\min _{1 \leq i \leq n}\left\{r_{i}(A)\right\}$

step 3. if $r_{\lambda}(A)>a_{\lambda \lambda}$

then compute $b_{i}=a_{i \lambda}+a_{\lambda \lambda}-r_{i}(A)$

$$
\begin{aligned}
& z_{i}=\frac{-b_{i}+\sqrt{b_{i}^{2}+4 a_{i \lambda}\left(r_{\lambda}(A)-a_{\lambda \lambda}\right)}}{2\left(r_{\lambda}(A)-a_{\lambda \lambda}\right)} \\
& 1 \leq i \leq n, i \neq \lambda
\end{aligned}
$$

find $\quad z=\min _{1 \leq i \leq n, i \neq \lambda}\left\{z_{i}\right\}$

compute $l b=z\left(r_{\lambda}(A)-a_{\lambda \lambda}\right)+a_{\lambda \lambda}$

else $l b=a_{\lambda \lambda}$

The complexity of the upper bound algorithm is analyzed

\begin{tabular}{|c|c|c|c|c|}
\hline & & calculations & $\begin{array}{l}\text { arithmetic } \\
\text { operations }\end{array}$ & comparisons \\
\hline 1 & & $\begin{array}{c}r_{i}(A)=\sum_{j=1}^{n} a_{i j} \\
1 \leq i \leq n\end{array}$ & $n(n-1)$ & \\
\hline 2 & $r_{\lambda}($ & $=\min \left\{r_{i}(A): 1 \leq i \leq n\right\}$ & & $n-1$ \\
\hline 3 & & if $r_{\lambda}(A)>a_{\lambda \lambda}$ & & 1 \\
\hline 4 & \multirow[t]{3}{*}{ then } & $\begin{array}{c}b_{i}=a_{i \lambda}+a_{\lambda \lambda}-r_{i}(A) \\
z_{i}=\frac{-b_{i}+\sqrt{b_{i}^{2}+4 a_{i \lambda}\left(r_{\lambda}(A)-a_{\lambda \lambda}\right)}}{2\left(r_{\lambda}(A)-a_{\lambda \lambda}\right)} \\
1 \leq i \leq n, i \neq \lambda\end{array}$ & $8 n-5$ & \\
\hline 5 & & $Z=\min _{1 \leq i \leq n, i \neq \lambda}\left\{Z_{i}\right\}$ & & $n-2$ \\
\hline 6 & & $l b=z\left(r_{\lambda}(A)-a_{\lambda \lambda}\right)+a_{\lambda \lambda}$ & 2 & \\
\hline
\end{tabular}
in the following.

\section{Complexity of lower bound algorithm I}




\begin{tabular}{|l|l|r|r|}
\hline 7 & else & $l b=a_{\lambda \lambda}$ & \\
\hline \multicolumn{3}{|c|}{ best case complexity } & $T(n)=n^{2}$ \\
\hline \multicolumn{3}{|c|}{ worst case complexity } & $T(n)=n^{2}+9 n-5$ \\
\end{tabular}

Remark 23. i) The quantities $r_{\lambda}(A)-a_{\lambda \lambda}, 2\left(r_{\lambda}(A)-a_{\lambda \lambda}\right)$ and $4\left(r_{\lambda}(A)-a_{\lambda \lambda}\right)$ required for computing the quantities $z_{i}$ are computed once.

ii) The computation of the quantity $r_{\lambda}(A)-a_{\lambda \lambda}$ required for computing the upper bound $l b$ has been already computed in previous calculations.

iii) Complexity.

The worst case appears, when $r_{\lambda}(A)>a_{\lambda \lambda}$. Then calculations 1, 2, 3, 4, 5, 6 are required. The worst case complexity is $T(n)=n^{2}+9 n-5 \in \mathrm{O}\left(n^{2}\right)$.

The best case appears when $r_{\lambda}(A)>a_{\lambda \lambda}$ does not hold. Then calculations $1,2,3,7$ are required. The best case complexity is $T(n)=n^{2} \in \Theta\left(n^{2}\right)$.

The complexity holds for $n \geq 2$. The case where $n=1$ is trivial; the algorithm is not implemented, since $l b=A$.

iv) Comment: The lower bound algorithm has two branches. The branch else computes the Frobenius' lower bound and the branch then computes a new (sharper) lower bound. It is obvious that steps 1 a nd 2 of lower bound algorithm compute the Frobenius' lower bound in time $T(n)=n^{2}-1 \in \mathrm{O}\left(n^{2}\right)$. Hence, the gain of computing a sharper lower bound than the Frobenius' lower bound costs computation time $T(n)=9 n-4 \in \mathrm{O}(n)$, which is derived by subtracting the Frobenius' complexity from the worst case complexity.

The algorithm for computing the upper bound of the spectral radius of a nonnegative matrix results from Lemma 1, Definition 2, Theorem 9 a nd Remark 10 a nd is summarized in the following.

\section{upper bound algorithm II}

\section{input: nonnegative matrix $A$}

output: upper bound $u b$ of the spectral radius of $A$

step 1. compute $r_{i}(A)=\sum_{j=1}^{n} a_{i j}, 1 \leq i \leq n$

step 2. find $r_{\mu}(A)=\max _{1 \leq i \leq n}\left\{r_{i}(A)\right\}$

step 3. if $r_{\mu}(A)>a_{\mu \mu}$

$$
\begin{aligned}
& \text { then compute } c_{j}=a_{j \mu}+a_{\mu \mu}-r_{j}(A) \\
& \qquad \begin{aligned}
w_{j} & =\frac{-c_{j}+\sqrt{c_{j}^{2}+4 a_{j \mu}\left(r_{\mu}(A)-a_{\mu \mu}\right)}}{2\left(r_{\mu}(A)-a_{\mu \mu}\right)} \\
1 \leq j \leq n, j \neq \mu & \\
w & =\max _{1 \leq j \leq n, j \neq \mu}\left\{w_{j}\right\} \\
\text { find } \quad u b & =w\left(r_{\mu}(A)-a_{\mu \mu}\right)+a_{\mu \mu} \\
\text { compute } u b &
\end{aligned} \\
& \text { else } u b=a_{\mu \mu}
\end{aligned}
$$

\begin{tabular}{|c|c|c|c|c|}
\hline & \multicolumn{2}{|r|}{ calculations } & $\begin{array}{l}\text { arithmetic } \\
\text { operations }\end{array}$ & comparisons \\
\hline 1 & & $\begin{array}{c}r_{i}(A)=\sum_{j=1}^{n} a_{i j} \\
1 \leq i \leq n\end{array}$ & $n(n-1)$ & \\
\hline 2 & \multicolumn{2}{|c|}{$r_{\mu}(A)=\max \left\{r_{i}(A): 1 \leq i \leq n\right\}$} & & $n-1$ \\
\hline 3 & \multicolumn{2}{|r|}{ if $r_{\mu}(A)>a_{\mu \mu}$} & & 1 \\
\hline 4 & \multirow[t]{3}{*}{ then } & $\begin{array}{c}c_{j}=a_{j \mu}+a_{\mu \mu}-r_{j}(A) \\
w_{j}=\frac{-c_{j}+\sqrt{c_{j}^{2}+4 a_{j \mu}\left(r_{\mu}(A)-a_{\mu \mu}\right)}}{2\left(r_{\mu}(A)-a_{\mu \mu}\right)} \\
1 \leq j \leq n, j \neq \mu\end{array}$ & $8 n-5$ & \\
\hline 5 & & $w=\max _{1 \leq j \leq n, j \neq \mu}\left\{w_{j}\right\}$ & & $n-2$ \\
\hline 6 & & $u b=w\left(r_{\mu}(A)-a_{\mu \mu}\right)+a_{\mu \mu}$ & 2 & \\
\hline 7 & else & $u b=a_{\mu \mu}$ & & \\
\hline \multicolumn{3}{|c|}{ best case complexity } & \multicolumn{2}{|c|}{$T(n)=n^{2}$} \\
\hline \multicolumn{3}{|c|}{ worst case complexity } & \multicolumn{2}{|c|}{$T(n)=n^{2}+9 n-5$} \\
\hline
\end{tabular}

The complexity of the upper bound algorithm is analyzed in the following.

\section{Complexity of upper bound algorithm II}

Remark 24. i) The quantities $r_{\mu}(A)-a_{\mu \mu}, 2\left(r_{\mu}(A)-a_{\mu \mu}\right)$ and $4\left(r_{\mu}(A)-a_{\mu \mu}\right)$ required for computing the quantities $w_{j}$ are computed once.

ii) The computation of the quantity $r_{\mu}(A)-a_{\mu \mu}$ required for computing the upper bound $u b$ has been already computed in previous calculations.

iii) Complexity.

The worst case appears, when $r_{\mu}(A)>a_{\mu \mu}$. Then calculations $1,2,3,4,5,6$ are required. The worst case complexity is $T(n)=n^{2}+9 n-5 \in \mathrm{O}\left(n^{2}\right)$.

The best case appears when $r_{\mu}(A)>a_{\mu \mu}$ does not hold. Then calculations $1,2,3,7$ are required. The best case complexity is $T(n)=n^{2} \in \Theta\left(n^{2}\right)$.

The complexity holds for $n \geq 2$. The case where $n=1$ is trivial; the algorithm is not implemented, since $u b=A$.

iv) Comment: The upper bound algorithm has two branches. The branch else computes the Frobenius' upper bound and the branch then computes a new (sharper) upper bound. It is obvious that steps 1 a nd 2 of upper bound algorithm compute the Frobenius' upper bound in time $T(n)=n^{2}-1 \in \mathrm{O}\left(n^{2}\right)$. Hence, the gain of computing a sharper upper bound than the Frobenius' upper bound costs computation time $T(n)=9 n-4 \in \mathrm{O}(n)$, which is derived by subtracting the Frobenius' complexity from the worst case complexity.

The algorithm for computing the new lower bound of the spectral radius of a nonnegative matrix results from the 
lower bound algorithm and the Proposition 14 and is summarized in the following.

\section{new lower bound algorithm III}

input: nonnegative matrix $A$

output: upper bound $l b$ of the spectral radius of $A$

step 1. compute $r_{i}(A), 1 \leq i \leq n$

step 2. find $r_{\lambda}(A)$

step 3. if $r_{\lambda}(A)>a_{\lambda \lambda}$

then compute $b_{i}, z_{i}, 1 \leq i \leq n, i \neq \lambda$

find $\quad z=\min _{1 \leq i \leq n, i \neq \lambda}\left\{z_{i}\right\}$

compute $l b=z\left(r_{\lambda}(A)-a_{\lambda \lambda}\right)+a_{\lambda \lambda}$

else $l b=a_{\lambda \lambda}$

construct submatrix $\tilde{A}$

of dimension $m \times m, 1 \leq m \leq n-1$

compute $r_{i}(\tilde{A}), \quad 1 \leq i \leq m$

find $r_{\tilde{\lambda}}(\tilde{A})$

if $r_{\tilde{\lambda}}(\tilde{A})>r_{\lambda}(A)$

then compute $\tilde{b}_{i}, \tilde{z}_{i}, 1 \leq i \leq m, i \neq \tilde{\lambda}$

find $\tilde{z}=\min _{1 \leq i \leq m, i \neq \tilde{\lambda}}\left\{\tilde{z}_{i}\right\}$

compute $n l b=\tilde{z}\left(r_{\tilde{\lambda}}(\tilde{A})-\tilde{a}_{\tilde{\lambda} \tilde{\lambda}}\right)+\tilde{a}_{\tilde{\lambda} \tilde{\lambda}}$

$l b=n l b$

Remark 25. In fact, the new lower bound algorithm computes the lower bound using the lower bound algorithm for the matrix $A$ or for the submatrix $\tilde{A}$, if needed.

Due to the fact that the dimension of the submatrix $\tilde{A}$ is less than the dimension of the matrix $A$, both the new lower bound algorithm III and the lower bound algorithm I have quadratic time worst case complexity $\mathrm{O}\left(n^{2}\right)$.

\section{CONCLUSIONS}

In this paper, a new lower bound and a new upper bound for the spectral radius of a nonnegative matrix are proposed. The main result of this paper is that the proposed upper and lower bounds are always sharper or equal to the wellestablished Frobenius' bounds.

The conditions under which the new bounds are sharper than the Frobenius' ones are determined. Clarifying examples are given in order to highlight the sharpness of the proposed bounds in comparison with the Frobenius' bounds.

An application in stability of SISO linear discrete time systems is presented and the stability of the systems is investigated.

The algorithms which implement the computation of the proposed bounds are presented and analyzed. The proposed bounds are computed with complexity $\mathrm{O}\left(n^{2}\right)$. This is very important especially in the case of large matrix dimension.

\section{REFERENCES}

[1] M. Adam, D. Aggeli and Aik. Aretaki, Some new bounds on the spectral radius of nonnegative matrices, AIMS Mathematics, v. 5(1), (2019), 701-716

[2] M. Adam, N. Assimakis and G. Tziallas, Generalized k,m-step Fibonacci sequences and matrices, Proceedings of the 12th International Conference of AHA-Algebraic Hyperstructures and its Applications, Int. J. of Algebraic Hyperstructures and Its Applications, v. 2(1), (2015), 125-134.

[3] B.D.O. Anderson and J.B. Moore, Optimal Filtering, Dover Publications, 2005.

[4] F. Babouklis, M. Adam and N. Assimakis, Bounds on the spectral radius of nonnegative matrices, Proceedings International Conference on Mathematics and Computers in Science and Engineering (MACISE), 2020, 51-55, DOI 10.1109/MACISE49704.2020.00016.

[5] B. Cantó, R. Cantó and S. Kostova, Stabilization of Positive Linear Discrete-Time Systems by Using a Brauer's Theorem, The Scientific World Journal, v. 2014, Article ID 856356, 6 pages, http://dx.doi.org/10.1155/2014/856356, 2014.

[6] B. Cantó, C. Coll and E. Sánchez, On stability and reachability of perturbed positive systems, Advances in Difference Equations, v. 296, (2014), 1-11,

http://www.advancesindifferenceequations.com/content/2014/1/296

[7] F. Duan and K. Zhang, An algorithm of diagonal transformation for Perron root of nonnegative irreducible matrices, Applied Mathematics and Computation, v. 175, (2006), 762-772.

[8] X. Duan and B. Zhou, Sharp bounds on the spectral radius of a nonnegative matrix, Linear Algebra and its Applications, v. 439, (2013), 2961-2970.

[9] E. Fornasini and M.E. Valcher, Reachability of a class of discretetime positive switched systems, v. SIAM J. Control Optim., v. 49, (2011), 162-184.

[10] M. Fu, Pole placement via static output feedback is NP-hard, Institute of Electrical and Electronics Engineers. Transactions on Automatic Control, v. 49(5), (2004), 855-857.

[11] R.A. Horn and C.R. Johnson, Matrix Analysis, Cambridge University Press, Cambridge, second edition, 2013.

[12] D. Laschov and M. Margaliot, Controllability of Boolean control networks via the Perron-Frobenius theory, Automatica, v. 48(6), (2012), 1218 - 1223

[13] Cui-Xia $\mathrm{Li}$ and Shi-Liang Wu, Some comparison results with new effective preconditioners for L-matrices, WSEAS Transactions on Mathematics, v. 13(8), (2014), 314-323.

[14] H. Lin and B. Zhou, On sharp bounds for spectral radius of nonnegative matrices, Linear and Multilinear Algebra, v. 65(8), (2017), 1554-1565.

[15] A. Melman, Upper and lower bounds for the Perron root of a nonnegative matrix, Linear and Multilinear Algebra, v. 61(2) (2013), 171-181.

[16] H. Minc, Nonnegative Matrices, John Wiley and Sons Inc., New York, 1988

[17] El-S. M.E. Mostafa, A.W. Aboutahoun and F.F.S. Omar, On the Solution of the Eigenvalue Assignment Problem for Discrete-Time Systems, Journal of Applied Mathematics, v. 2017, Article ID 7256769, (2017), 12 pages.

[18] R. Xing and B. Zhou, Sharp bounds for the spectral radius of nonnegative matrices, Linear Algebra and its Applications, v. 449, (2014), 194-209.

[19] Mengmeng Xu, Junguo Jia and Peng Zhao, The Progress for Stability of Essential and Critical Spectra of Perturbed C0-semigroups and its Applications to Models of Transport Theory, WSEAS Transactions on Mathematics, v.11(6), (June, 2012), 558-566.

[20] P. Walters, An introduction to ergodic theory, New York (NY), Springer-Verlag, 1982.

[21] C. Wen and T.Z. Huang, A modified algorithm for the Perron root of a nonnegative matrix, Appl. Math. Comput., v. 217, (2011), 44534458 .

\section{Creative Commons Attribution License 4.0 (Attribution 4.0 International, CC BY 4.0)}

This article is published under the terms of the Creative Commons Attribution License 4.0

https://creativecommons.org/licenses/by/4.0/deed.en US 